\title{
A New Algorithm of Self Organization in Wireless Sensor Network
}

\author{
Hemanta Kumar KALITA, Avijit KAR \\ Department of Computer Engineering, Jadavpur University, Kolkata, India \\ Email: hemanta91@yahoo.co.in,avijit.kar@gmail.com \\ Received September 6, 2009; revised October 10, 2009; accepted October 13, 2009
}

\begin{abstract}
Self organization is one of the most important characteristics in an Ad-hoc Sensor Network. Thousands of Sensors are deployed in a geographical area randomly without considering the location factor. After deployment, sensors are to self organize themselves to form a network of their own. How well the network is formed determines the life of the whole network as well as the quality of data transmission. Self organization based on clustering has proven to be very useful in this regard. Since hierarchical clustering reduces energy consumption by routing data from one node to another. In this paper, we discuss a new algorithm for self organization of sensors deployed in a geographical area. The algorithm forms clusters of sensors by ordering them using a unique triangulation method. This algorithm not only considers all sensors but also groups them so that their inherent clustering property is preserved.
\end{abstract}

Keywords: Self-Organization, Wireless Sensor Network, OPTICS

\section{Introduction}

Sensor network is a computer network of many spatially distributed devices using sensors to monitor conditions at different locations such as temperature, sound, vibration, pressure, motion or pollutants. Usually these devices are small and inexpensive, so that they can be produced and deployed in large numbers, and so their resources in terms of energy, memory, computational speed and bandwidth are severely constrained. Each device is equipped with a radio transceiver, a small micro controller and an energy source, usually a battery. The devices use each other to transport data to a monitoring computer [1]. Data from the sensors is aggregated and analyzed by a computer outside the network. This computer is connected to the network by a special network node called gateway node [2]. Sensor networks involve three areas: sensing, communications and computation. In other words, hardware, software and algorithms [3,4].

An Ad hoc Sensor Network is a self-organizing multihop wireless network, which relies neither on fixed infrastructure nor on predetermined connectivity [5]. This property enables rapid deployment without precise prior knowledge of the coverage area of interest - hence serves well for situations lacking fixed infrastructure or high risk, e.g., in military communications, disaster manage- ment, law enforcement, and etc [6]. In this paper we propose a new algorithm for Self Organization of Sensors in an Ad hoc Sensor Network [7].

Remainder of the paper is divided into four sections. In Section 2, we discuss related work. In Section 3, we propose a new algorithm for self organization of sensor nodes in an ad hoc sensor network. Section 4 is for the experimental results and Section 5 finally concludes the paper.

\section{Related Work}

In [8] proposes a Self Organizing Sensor (SOS) network based on an intelligent clustering algorithm which does not require many user defined parameters and random selection to form clusters. The proposed SOS algorithm can reduce the number of cluster heads.

[9] discusses Ultra-wideband (UWB) technology. UWB has proven to be useful in short range, high data rate, robust, and low power communications. These features can make UWB systems ideal candidates for reliable data communications between nodes of a wireless sensor network (WSN). However, the low powered UWB pulses can be significantly degraded by channel noise, inter-node interference, and intentional jamming. [9] presents a novel interference suppression technique for UWB based WSNs that promises self-organization in 
terms of power conservation, scalability, and channel estimation for the entire distributed network.

[5] approaches to achieve an emerging distributed perception of the sensed environment in real-time through clustering. Research in classifying and recognizing complex concepts has been directing its focus increasingly on distributed sensing using a large amount of sensors. The Colossal amount of sensor data often obstructs traditional algorithms in centralized approaches, where all sensor data is directed to one central location to be processed. Spreading the processing of sensor data over the network seems to be a promising option, but distributed algorithms are harder to inspect and evaluate. By using self-sufficient sensor boards with short-range wireless communication capabilities, [5] are exploring this.

Bio-inspired communication methodologies promise to enable more scalable selforganizing network infrastructures. Especially in the area of mobile ad hoc sensor networks, such solutions are required in order to qualify them for simplified development and deployment based on autonomously evolving mechanisms to work on global tasks, i.e. to show an emergent behavior. [10] introduce their ongoing research of Autonomic Networking focused on the developments on efficient data dissemination in sensor networks. A particular example of how to study biological processes and to adapt the results in communication networks, the feedback loop mechanism, depicts the potentials of this research area.

[11] develop an energy-aware self organized routing algorithm for the networking of simple battery powered wireless micro-sensors (as found, for example, in security or environmental monitoring applications). In these networks, the battery life of individual sensors is typically limited by the power required to transmit their data to a receiver or sink. Thus effective network routing algorithms allow reducing this power and extending both the lifetime and the coverage of the sensor network as a whole. However, implementing such routing algorithms with a centralized controller is undesirable due to the physical distribution of the sensors; their limited localization ability and the dynamic nature of such networks (given that sensors may fail, move or be added at any time and the communication links between sensors are subject to noise and interference). Against this background, [11] present a distributed mechanism that enables individual sensors to follow locally selfish strategies, which, in turn, result in the self organization of a routing network with desirable global properties [12,13].

\section{Proposed Algorithm}

The idea of a new algorithm for self organization of sen-

\footnotetext{
${ }^{1}$ The terms defined here are to make it suitable for describing the new algorithm. We do not know, if exactly the same definition exists for these or not.
}

sor nodes in an Ad hoc Sensor Network stems from our original work on spatial clustering. Again, from our study of routing issues of wireless sensor network [14], we have found that-cluster based method has lots of advantages too. A great spatial clustering algorithm called OPTICS [15-18] has noble idea of ordering point data to get arbitrary shaped cluster. Our new algorithm is primarily based on OPTICS. But, the new algorithm, orders sensor nodes by an unique method of triangulation $[19,20]$. When the ordering procedure is over, all the sensor nodes form a network of their own and become member of a cluster.

Our goal is to design an algorithm for self organization of sensor nodes in an Adhoc Sensor Network. The algorithm should be able to connect all sensor nodes. At the same time, sensor nodes should self organize in such a way that resource usage, for example, battery power is minimum [21]. This is possible only when the inherent clusters are detected by the algorithm [22].

The new algorithm self organizes sensor nodes by forming cluster. Clusters are formed by an ordering procedure which has similarity with the OPTICS Algorithm. In our approach we have assumed that a better quality cluster means sensor nodes in that cluster use minimal resource for sensing, communicating and receiving data.

\subsection{Definitions}

We define some terminologies which will be used while discussing our Algorithm ${ }^{1}$.

Definition 1 Point: A Point is a sensor node deployed in a geographical area. In our algorithm, we assume that the Point can communicate with another Point using wireless media.

Definition 2 Edge: An Edge $\overline{A B}$ connects two Points $A$ and $B$. Weight of an Edge, is the time required to send a request from $A$ to $B$ and getting response from $B$ to $A$ [23]. Such three edges connecting three Points $A, B$ and $C$ form a triangle.

Definition 3 minTriangle: A triangle $\mid A B C$ formed by three Points $A, B$ and $C$ is a minTriangle if the summation of weights of the three edges $\overline{A B}, \overline{B C}$ and $\overline{C A}$ is minimum out of all possible triangles considering $\overline{A B}$ as one Edge in the geographical area.

Definition 4 Live Edge: This is an Edge whose two Points will be the two Points of the next minTriangle

\subsection{The Algorithm}

INPUT: A Set of Points spread on a geographical area. OUTPUT: Clusters of Points connected to each other based on the Weights.

Step-1: Choose a Point, A randomly. 
Step-2: Find another Point, B such that is minimum. Mark Points $\mathrm{A}$ and $\mathrm{B}$ as connected. Initially, $\overline{A B}$ is a live edge.

Step-3: Find a new point $C$ such that triangle $\mid A B C$ is a minTriangle. Also, mark Point, $\mathrm{C}$ as connected.

Step-4: Choose a new live edge out of $\overline{A C}$ and $\overline{B C}$. Step-5: If then $\overline{A C}$ is the new live edge. $\overline{A B}=\overline{A C}$. Step-6: Else, $\overline{B C}$ is the new live edge. $\overline{A B}=\overline{B C}$.

Step-7 Repeat steps 3-6 until all Points are Connected.

\subsection{Analysis}

The self organization algorithm that we have described is very simple. Time complexity is $O\left(n^{2}\right)$, where $n$ is the total number of Points. This can be further reduced if we make the algorithm distributed.

\section{Experimental Results}

The algorithm is implemented using Java and experimented on Linux 8.0 platform. We have used synthetic Point $^{2}$ data sets. Figure 1 shows initial isolated Points.

In Figure 2, the algorithm finds the first minTriangle.

In Figure 3, we see how the algorithm proceeds by finding minTriangles step by step.

Finally, in Figure 4, we see how the Points get connected. It is clear from Figure 4 that Points are connected first in their own cluster. Then one cluster gets connected to another cluster.

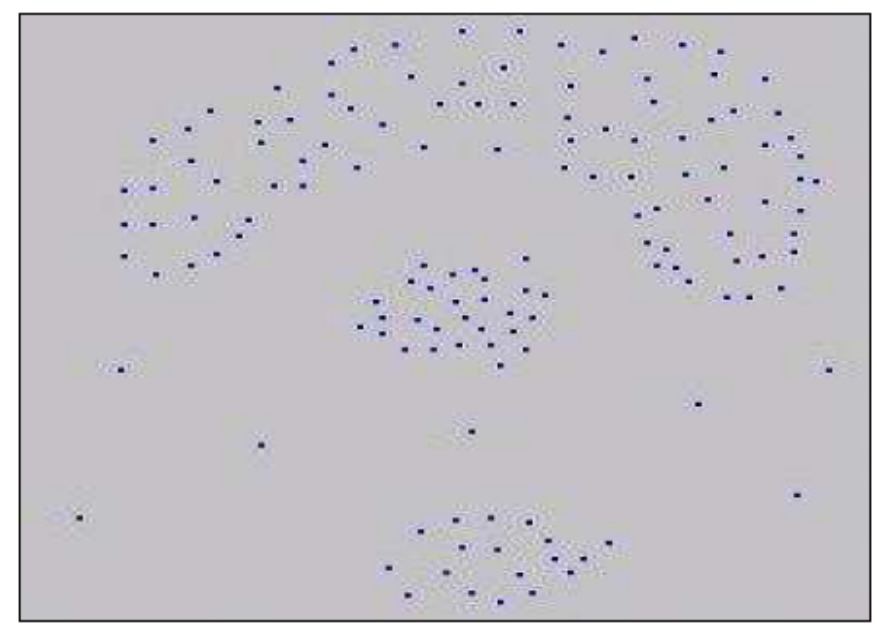

Figure 1. Input-A set of points.

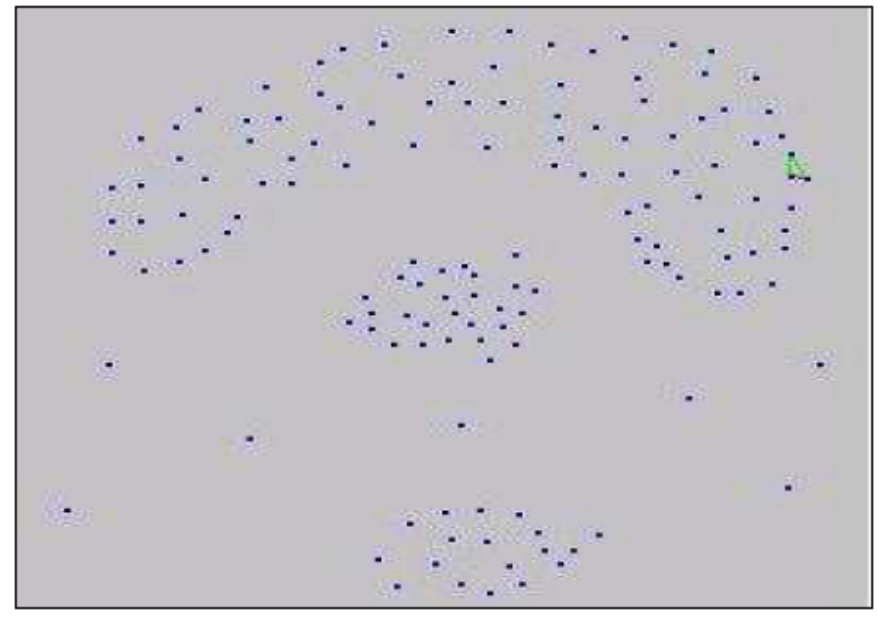

Figure 2. First minTriangle.

${ }^{2}$ Note that a Point is a Sensor Node. 


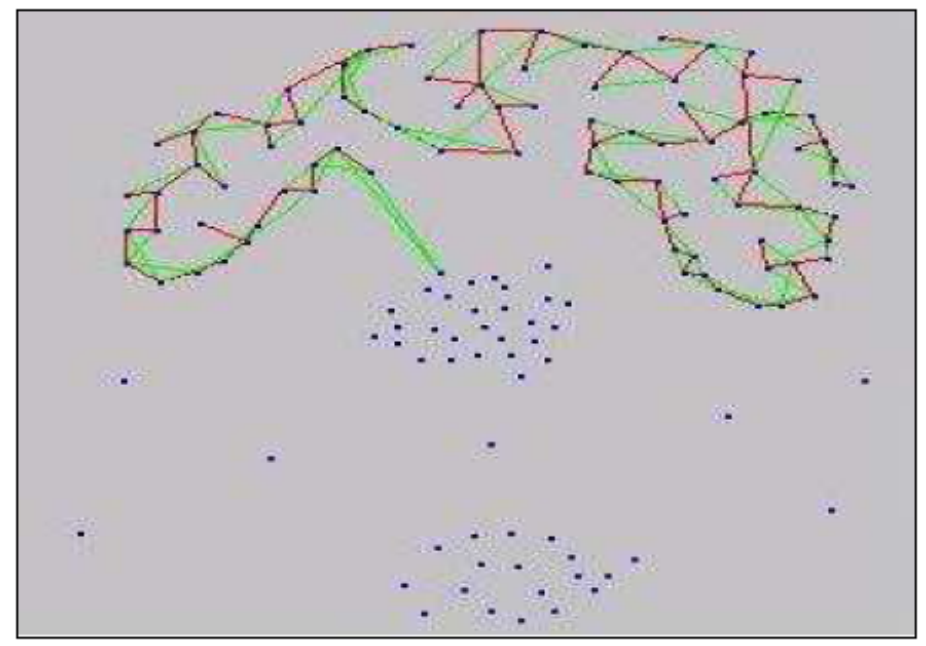

Figure 3. After finding few triangles.

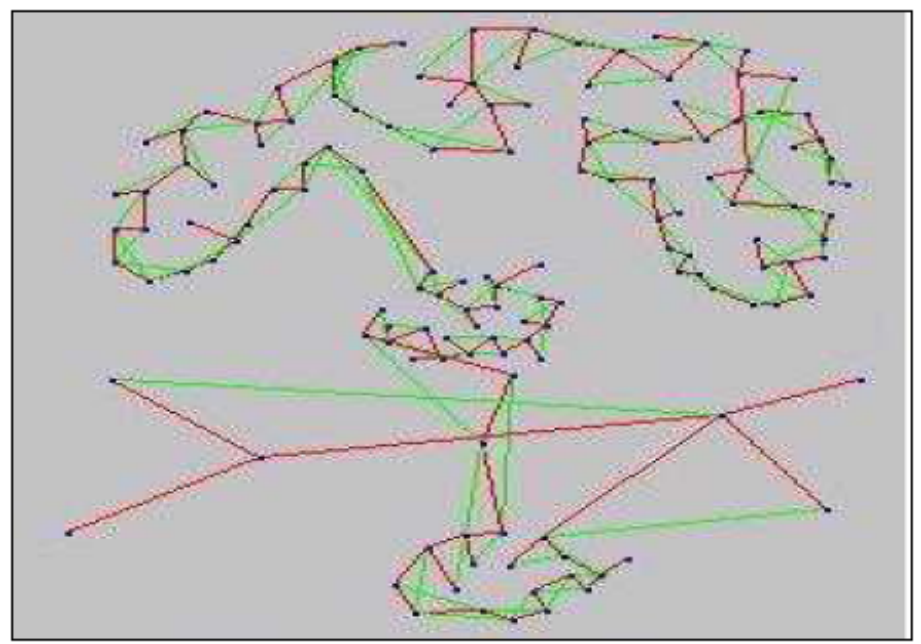

Figure 4. Output-Triangulation of the whole points.

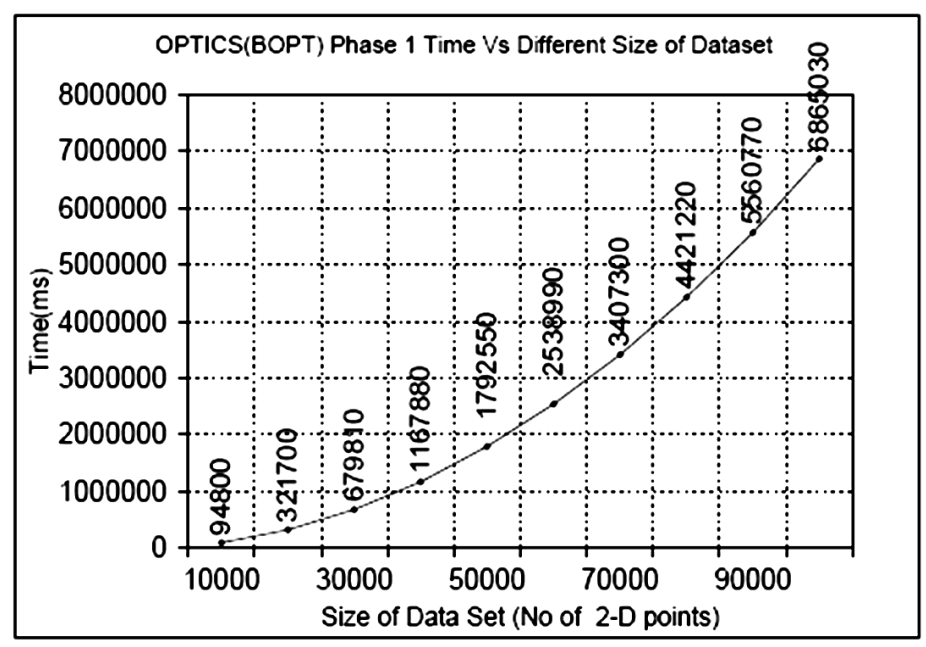

Figure 5. Scalability of the proposed algorithm. 
We have performed scalability test of the algorithm in a machine with CPU speed $2.0 \mathrm{GHz}$ and memory size $128 \mathrm{MB}$. In this regard, ten experiments were done by choosing size of Point data set from 10000 to 100000 . Data sets are generated using randomizer. Outcome of these experiments are shown in the graph of Figure 5. From the graph we see that the algorithm is scalable for large Point data set.

\section{Conclusions}

In this paper, we have proposed a new algorithm for self organization of sensors in an Ad hoc Sensor Network based on OPTICS [14] and OPTICS (BOPT) [18]. We also demonstrated how the algorithm works. And, scalability is not too high but moderate. This can be improved further by making the algorithm distributed. Also, we did not consider the security [24,25] aspect while discussing the algorithm. This is part of our ongoing research.

\section{References}

[1] M. B. Srivastava and C. Schurgers, "Energy efficient routing in wireless sensor networks,” 2000.

[2] D. Ganeshan, A. Cerpa, W. Ye, Y. Yu, J. Zhao, and D. Estrin, "Networking issues in wireless sensor network," 2003.

[3] H. Kumar and K. R. Gurung, "Routing issues in wireless sensor network," Network, Data Mining \& AI, Narosa Pub, ISBN 81-7319-755-5, 2006.

[4] J. Ibriq and I. Mahgoub, "Cluster based routing in wireless sensor networks: issues and challenges,” SPECTS’04, No. 759, pp. 1, 2004.

[5] K. V. Laerhoven, E. Catterall, and M. Strohbach, "Self-organization in ad hoc sensor networks: An empirical study,” in Artificial Life VIII, Standish, Abbass, Bedau (eds) (MIT Press), pp. 260-263, 2002.

[6] R. Batta, R. Nagi, A. Joshi, and N. Mishra, “Ad hoc sensor network topology design for distributed fusion: A mathematical programming approach, ” 2003.

[7] R. Wattenhofer, "Algorithms for ad hoc and sensor networks,” 2005.

[8] A. Abraham, K. Shin, and S. Y. Han, "Self organizing sensors by minimization of cluster heads using intelligent clustering,” 2004.

[9] A. Spiridon, F. Nekoogar, and F. Dowla, "Self organiza- tion of wireless sensor networks using ultra-wideband radios,” UCRL-CONF-205469, 2004.

[10] G. Fuchs, R. German, F. Dressler, and B. Krger, "Self-organization in sensor networks using bio-inspired mechanisms,” 2005.

[11] E. David, A. Rogers, and N. R. Jennings, "Self-organized routing for wireless micro-sensor networks,” 2004.

[12] V. Ailawadhi, K. Sohrabi, J. Gao, and G. J. Pottie, UCLA, "Protocols for self-organization of a wireless sensor network,” IEEE Personal Communications, 2000.

[13] D. Starobinski and R. Krishnan, "Message-efficient selforganization of wireless sensor networks," 2001.

[14] H. K. Kalita, "Securing wireless sensor network,” NCS2005, Allied Pub, ISBN 81-7764-931-0, 2005.

[15] M. Ankerst, M. M. Breunig, H. P. Kriegel, and J. Sander, "Optics: Ordering points to identify the clustering structure,” In Proceedings of ACM SIGMOD’99, Institute of Computer Science, University of Munich, Germany, 1999.

[16] J. Han and M. Kamber, "Data mining: Concepts and techniques,” Morgan Kaufmann, San Francisco, CA, USA, 2003.

[17] Z. Huang, "Clustering large data sets with mixed numeric and categorical values."

[18] E. Kolatch, "Clustering algorithms for spatial databases: A survey,” 2001.

[19] H. Kalita, "A new algorithm for ordering of points to identify clustering structure based on perimeter of triangle: Optics(bopt),” Master's thesis, Department of Computer Sc \& IT, Tezpur University, Assam(India), 2004.

[20] C. Eldershaw and M. Hegland, "Cluster anaysis using triangulation,” Computational Techniques and Applications World Scientific, 1997.

[21] D. Estrin, Y. Yu, and R. Govindan, "Geographical and energy aware routing: A recursive data dissemination protocol for wireless sensor networks,” 2001.

[22] G. Gupta and M. Younis, "Load-balanced clustering in wireless sensor networks,” 2003.

[23] J. F. Roddick, K. Hornsby, and D. d Vries, “A unifying semantic distance model for determining the similarity of attribute values," 2003.

[24] W. S. Shi, J. P. Walters, Z. Q. Liang, and V. Chaudhary, "Wireless sensor network security: A survey," Security in Distributed, Grid, and Pervasive Computing, Yang Xiao, Auerbach Publications, CRC Press, 2006.

[25] C. Karlof and D. Wagner, "Secure routing in wireless sensor networks: Attacks and countermeasures,” Ad Hoc Networks 1 (2003) 293315, 2003. 